\title{
BEHAVIOR OF THE EULER SCHEME WITH DECREASING STEP IN A DEGENERATE SITUATION
}

\author{
VINCENT LEMAIRE ${ }^{1}$
}

\begin{abstract}
The aim of this short note is to study the behavior of the weighted empirical measures of the decreasing step Euler scheme of a one-dimensional diffusion process having multiple invariant measures. This situation can occur when the drift and the diffusion coefficient are vanish simultaneously.
\end{abstract}

Mathematics Subject Classification. 60H10, 65C30, 37M25.

Received April 3, 2006. Revised October 16, 2006.

\section{INTRODUCTION AND FRAMEWORK}

First, let us recall some results about the behavior of a one-dimensional diffusion process. Let $I=] l, r[$ denote an open (non-trivial) interval of the real line $\mathbf{R}$. We consider the following stochastic differential equation

$$
\mathrm{d} X_{t}=b\left(X_{t}\right) \mathrm{d} t+\sigma\left(X_{t}\right) \mathrm{d} B_{t},
$$

where $X_{0}$ is a random variable taking values in $I$ and $\left(B_{t}\right)_{t \geqslant 0}$ a standard Brownian motion on $\mathbf{R}$. We assume that $b$ and $\sigma$ are continuous functions on $\bar{I}$ taking values in $\mathbf{R}$, and that $\sigma$ is not degenerate on $I$ i.e. $\forall x \in I$, $\sigma^{2}(x)>0$. Then there exists a unique solution $\left(X_{t}\right)_{t \geqslant 0}$ adapted to the completed Brownian filtration, such that $t \mapsto X_{t}$ is continuous on $\left[0, \zeta\left[\right.\right.$, where $\zeta=\inf \left\{t \geqslant 0, X_{t}=l\right.$ or $\left.X_{t}=r\right\}$ is the explosion time of the diffusion.

The classification of one-dimensional diffusion processes is due to Feller, in particular in [1] and [2]. An overview and a comparison with the Russian terminology are given in [4]. In this classification, there is essentially two concepts: "attractivity" and "attainability" to determine the behavior of the diffusion in a neighborhood of a boundary point. The notion of attractivity of a boundary point ( $l$ or $r$ ) is defined using the scale function. The scale function $p$ is a strictly increasing function defined up to an affine transformation and such that the process $\left(p\left(X_{t}^{\zeta}\right)_{t}\right)_{t \geqslant 0}$ is a local martingale. In our framework ( $\sigma$ not degenerated on $\left.I\right), p$ is in $\mathcal{C}^{2}(I, \mathbf{R})$ and satisfies $\mathcal{A} p=0$ where $\mathcal{A}$ is the infinitesimal generator. The attainability of a boundary point is defined using the speed measure of the process $i . e$. the measure with density $m=\frac{2}{\sigma^{2} p^{\prime}}$ with respect to the Lebesgue measure.

A boundary point $\Delta(\Delta=l$ or $\Delta=r)$ is said to be attractive if $\lim _{b \rightarrow \Delta}|p(b)|<+\infty$ and repulsive if not. From a probabilistic point of view, if $\Delta$ is an attractive boundary point, then for all $a \in I$ and all $x$ in the open

Keywords and phrases. One-dimensional diffusion process, degenerate coefficient, invariant measure, Euler scheme.

1 Laboratoire d'Analyse et de Mathématiques Appliquées, UMR 8050, Université de Marne-la-Vallée, 5 boulevard Descartes, Champs-sur-Marne, 77454 Marne-la-Vallée Cedex 2, France; Vincent.Lemaire@univ-mlv.fr

(c) EDP Sciences, SMAI 2007 
interval with endpoints $a$ and $\Delta$ we have

$$
\mathbf{P}_{x}\left[\lim _{\substack{b \rightarrow \Delta \\ b \in I}} T_{b} \leqslant T_{a}\right]>0
$$

where $T_{a}$ denotes the hitting time of the one-point set $\{a\}$ i.e. $T_{a}=\inf \left\{t \leqslant 0, X_{t}=a\right\}$. If $\Delta$ is attractive then $\Delta$ is attainable if and only if $\mathbf{P}_{x}\left[T_{\Delta}<+\infty\right]>0$. More generally, we say that a boundary point is attainable if for all $a \in I$ and $x$ in the open interval of endpoints $a$ and $\Delta$ we have

$$
\lim _{b \rightarrow \Delta} \mathbf{E}_{x}\left[T_{b} \wedge T_{a}\right]<+\infty
$$

Furthermore, in this paper, we say that a repulsive boundary point $\Delta$ is strongly repulsive if for every $c \in I$ we have $\left|\int_{\Delta}^{c} m(y) \mathrm{d} y\right|<+\infty$. The speed measure is thus finite in a neighborhood of a strongly repulsive point. Using these definitions, we are able to establish the following classification of the ergodic behavior of the diffusion.

Theorem 1.1. We recall that $\zeta=\inf \left\{t \geqslant 0, X_{t}=l\right.$ or $\left.X_{t}=r\right\}$. Then

- if $l$ is attractive and $r$ is repulsive then $X_{t}^{\zeta} \stackrel{a . s}{\longrightarrow} l$;

- if $l$ and $r$ are attractive then

$$
\mathbf{P}\left[\lim _{t \rightarrow+\infty} X_{t}^{\zeta}=l\right]=1-\mathbf{P}\left[\lim _{t \rightarrow+\infty} X_{t}^{\zeta}=r\right]=\frac{p\left(r^{-}\right)-p\left(X_{0}\right)}{p\left(r^{-}\right)-p\left(l^{+}\right)}
$$

- if $l$ and $r$ are repulsive then the diffusion is recurrent and does not explode $(\zeta=+\infty$ a.s. $)$. More precisely

- if $l$ and $r$ are strongly repulsive (i.e. the speed measure is finite) then the diffusion is positive recurrent and $\nu_{t} \Rightarrow \nu$ a.s. where $\nu$ is the normalized speed measure. Moreover

$$
\forall f \in \mathbf{L}^{1}(\nu), \quad \frac{1}{t} \int_{0}^{t} f\left(X_{s}\right) \mathrm{d} s \longrightarrow \int_{\mathbf{R}} f(x) \nu(\mathrm{d} x) \quad \text { a.s. }
$$

- if $l$ is strongly repulsive and $r$ is (simply) repulsive then the diffusion is null recurrent and if the empirical measures are tight we have

$$
\frac{1}{t} \int_{0}^{t} \delta_{X_{s}} \mathrm{~d} s \Rightarrow \delta_{r} \quad \text { a.s. }
$$

- if $l$ and $r$ are not strongly repulsive then the diffusion is null recurrent, and if the empirical measures are tight then any weak limit of $\left(\frac{1}{t} \int_{0}^{t} \delta_{X_{s}} \mathrm{~d} s\right)_{t \geqslant 1}$ is a measure with support $\{l, r\}$.

The first two items are proved in [3] (Prop. 5.22). The third item is proven in Appendix A.

Let us now recall the link between the concepts of attractivity, repulsivity and strong repulsivity, and the Lyapunov functions. This link given by the following proposition is useful to study the ergodic behavior of the decreasing step Euler scheme. In the sequel, we will denote by $J_{\Delta} \subset I$ an open (non-trivial) interval included in $I$ with endpoint $\Delta$.

Proposition 1.2. Let $\Delta$ a boundary point of $I$.

(1) $\Delta$ is a repulsive boundary point of $I$ if and only if there exists a neighborhood $J_{\Delta} \subset I$ of $\Delta$ and a strictly monotone function $v \in \mathcal{C}^{2}\left(\bar{J}_{\Delta}, \mathbf{R}_{+}\right)$satisfying $v(\Delta)=0$, such that

$$
\forall x \in J_{\Delta}, \quad \mathcal{A} v(x) \geqslant \frac{1}{2} \sigma^{2}(x) \frac{\left(v^{\prime}(x)\right)^{2}}{v(x)},
$$


(2) $\Delta$ is a strongly repulsive boundary point of I if and only if there exists a neighborhood $J_{\Delta} \subset I$ of $\Delta$ and a strictly monotone function $v \in \mathcal{C}^{2}\left(\bar{J}_{\Delta}, \mathbf{R}_{+}\right)$having a minimum at $\Delta$, such that

$$
\exists \varepsilon>0, \quad \forall x \in J_{\Delta}, \quad \mathcal{A} v(x) \geqslant \frac{1}{2} \sigma^{2}(x) \frac{\left(v^{\prime}(x)\right)^{2}}{v(x)}+\varepsilon v(x),
$$

(3) $\Delta$ is an attractive boundary point of $I$ if and only if there exists a neighborhood $J_{\Delta} \subset I$ of $\Delta$ and a strictly monotone function $v \in \mathcal{C}^{2}\left(\bar{J}_{\Delta}, \mathbf{R}_{+}\right)$having a minimum at $\Delta$, such that

$$
\forall x \in J_{\Delta}, \quad \mathcal{A} v(x)<\frac{1}{2} \sigma^{2}(x) \frac{\left(v^{\prime}(x)\right)^{2}}{v(x)} .
$$

This proposition is proven in Appendix B.

Remark 1.3. If $\Delta$ is such that $b(\Delta)=\sigma(\Delta)=0$, then $\Delta$ is a critical point for the equation $u^{\prime}=b(u)$. It is worth noting that there exists a link between the nature of the critical point $\Delta$ for the ODE $u^{\prime}=b(u)$ and the nature of the boundary point $\Delta$ for the SDE.

Indeed, if $\Delta$ is a stable critical point then there exists a Lyapunov function $F \in \mathcal{C}^{2}$ such that $F^{\prime} b(u)<0$ for every $u$ in a neighborhood of $\Delta$. If $F^{\prime} / F$ is decreasing, the above proposition implies that $\Delta$ is an attractive boundary point for the SDE.

If $\Delta$ is an unstable point for the ODE $u^{\prime}=b(u)$, it may be repulsive, strongly repulsive or attractive for the SDE, as shown in Example 2.6.

Let us now suppose that the diffusion $\left(X_{t}\right)_{t \geqslant 0}$ on the real line has (at least) a point $\Delta$ such that $b(\Delta)=$ $\sigma(\Delta)=0$. In this situation we have

$$
\left.\left.\forall x \in]-\infty, \Delta\left[, \quad \mathbf{P}_{x}\left[X_{t} \in\right]-\infty, \Delta\right]\right]=1, \quad \text { and } \quad \forall x \in\right] \Delta,+\infty\left[, \quad \mathbf{P}_{x}\left[X_{t} \in[\Delta,+\infty[]=1 .\right.\right.
$$

In fact, the process $\left(X_{t}\right)_{t \geqslant 0}$ has an ergodic behavior in $\left.I_{1}=\right]-\infty, \Delta\left[\right.$ or in $\left.I_{2}=\right] \Delta,+\infty[$ according to the starting point $x$. The Euler scheme of this diffusion is not continuous and may a priori jump above the boundary point $\Delta$. In this note, we show that in some particular cases the boundary $\Delta$ becomes a border for the scheme after an almost surely finite time. This is the principal result given by Theorem 2.1. We next prove in Proposition 2.5 that the empirical measures of the scheme have the same behavior than the empirical measures of the diffusion. Finally, a numerical example is given to illustrate these results.

\section{BeHAVIOR OF THE EULER SCHEME WITH DECREASING STEP}

The Euler scheme $\left(X_{n}\right)_{n \geqslant 0}$ with decreasing step $\left(\gamma_{n}\right)_{n \geqslant 1}$ is defined as follows. Consider a positive sequence $\left(\gamma_{n}\right)_{n \geqslant 1}$ satisfying $\lim _{n} \sum_{k=1}^{n} \gamma_{k}=+\infty$ and denote $\Gamma_{n}=\sum_{k=1}^{n} \gamma_{k}$. The Euler scheme is the inhomogeneous Markov chain defined as

$$
X_{n+1}=X_{n}+\gamma_{n+1} b\left(X_{n}\right)+\sqrt{\gamma_{n+1}} \sigma\left(X_{n}\right) U_{n+1}
$$

with $\left(U_{n}\right)_{n \geqslant 1}$ a real white noise $i$.e. a sequence of $i . i$.d. random variables such that $\mathbf{E}\left[U_{1}\right]=0$ and $\operatorname{var}\left(U_{1}\right)=1$. Furthermore, we assume that $U_{1}$ is a generalized Gaussian (cf. [9]) i.e. such that

$$
\exists \kappa>0, \forall \theta \in \mathbf{R}, \quad \mathbf{E}\left[\exp \left(\theta U_{1}\right)\right] \leqslant \exp \left(\frac{\kappa|\theta|^{2}}{2}\right) .
$$

For example $U_{1}$ is a standard Gaussian or a Bernoulli random variable. A consequence of the generalized Gaussian property is the following

$$
\forall a \geqslant 0, \quad \mathbf{P}\left[\left|U_{1}\right| \geqslant a\right] \leqslant \exp \left(-\frac{a^{2}}{2 \kappa}\right) .
$$


We consider a diffusion $\left(X_{t}\right)_{t \geqslant 0}$ on the real line with $b$ and $\sigma$ continuous on $\mathbf{R}$. Moreover we assume that $b$ and $\sigma$ have sublinear growth i.e.

$$
\exists C_{b}>0, \quad|b|^{2} \leqslant C_{b}(1+|x|), \quad \text { and } \exists C_{\sigma}>0, \quad|\sigma|^{2} \leqslant C_{\sigma}(1+|x|) .
$$

In the sequel $\Delta$ denotes a finite point of $\mathbf{R}$ such that $b(\Delta)=\sigma(\Delta)=0$, and $J_{\Delta}$ denotes an open interval with endpoint $\Delta\left(J_{\Delta}=\right] \Delta, \Delta+\varepsilon[$ if $\Delta$ is a left endpoint of $I=] \Delta,+\infty\left[\right.$ and $\left.J_{\Delta}=\right] \Delta-\varepsilon, \Delta[$ if it is a right endpoint of $I=]-\infty, \Delta[)$.

\subsection{Euler scheme}

We prove the following theorem which gives the behavior of one trajectory of the Euler scheme $\left(X_{n}\right)_{n \geqslant 0}$ in this degenerate situation.

Theorem 2.1. We assume that $\sigma$ satisfies $\sigma(x) \neq 0$ for every $x \in]-\infty, \Delta[\cup] \Delta,+\infty[$ and that there exists $\left.\mathcal{U}_{\Delta}=\right] \Delta-\varepsilon, \Delta+\varepsilon\left[\right.$ with $\varepsilon>0$, and a convex function $v \in \mathcal{C}^{2}\left(\mathcal{U}_{\Delta}, \mathbf{R}_{+}\right)$satisfying $v(\Delta)=0$ and

$$
\forall x \in \mathcal{U}_{\Delta}, \quad\left(v^{\prime} b\right)(x) \geqslant 0 \quad \text { and } \quad \exists c_{\sigma}>0, \forall x \in \mathcal{U}_{\Delta}, \quad\left|\left(v^{\prime} \sigma\right)(x)\right| \leqslant c_{\sigma} v(x) .
$$

If the step sequence $\left(\gamma_{n}\right)_{n \geqslant 1}$ satisfies $\forall C>0, \sum_{n \geqslant 1} \exp \left(-\frac{C}{\gamma_{n}}\right)<+\infty$ then the Euler scheme jump above $\Delta a$ finite number of times i.e.

$$
\mathbf{P}\left[\exists n_{0} \geqslant 0, \forall n \geqslant n_{0}, X_{n} \in\right]-\infty, \Delta[]+\mathbf{P}\left[\exists n_{0} \geqslant 0, \forall n \geqslant n_{0}, X_{n} \in\right] \Delta,+\infty[]=1 .
$$

We first prove the following lemma.

Lemma 2.2. We assume there exists a convex function $v \in \mathcal{C}^{2}\left(\bar{J}_{\Delta}, \mathbf{R}_{+}\right)$satisfying $v(\Delta)=0$ and

$$
\forall x \in \bar{J}_{\Delta}, \quad\left(v^{\prime} b\right)(x) \geqslant 0 \quad \text { and } \quad \exists c_{\sigma}>0, \forall x \in J_{\Delta}, \quad\left|\left(v^{\prime} \sigma\right)(x)\right| \leqslant c_{\sigma} v(x) .
$$

Then, on the event $\left\{X_{n} \in \bar{J}_{\Delta}\right\}$

$$
\mathbf{P}\left[\Delta \in\left(X_{n}, X_{n+1}\right) \mid \mathcal{F}_{n}\right] \leqslant \exp \left(-\frac{1}{c_{\sigma}^{2} \gamma_{n+1}}\right) .
$$

Proof. We assume that $\Delta$ is a left endpoint of $I$ and we denote by $A_{n+1}$ the event $\left\{\Delta \in\left(X_{n}, X_{n+1}\right)\right\}$ (the geometric segment with endpoints $X_{n}$ and $\left.X_{n+1}\right)$. Since $v$ is continuous and $v(\Delta)=0$ it is clear that

$$
\begin{aligned}
A_{n+1} & \left.=\left\{\exists t \in[0,1], X_{n}+t\left(X_{n+1}-X_{n}\right)\right)=\Delta\right\}, \\
& \subset\left\{\exists t \in[0,1], v\left(X_{n}+t\left(X_{n+1}-X_{n}\right)\right)=0\right\} .
\end{aligned}
$$

Consider $t \in[0,1]$ such that $\Delta=X_{n}+t\left(X_{n+1}-X_{n}\right)$. As $v$ is $\mathcal{C}^{2}$ on $\bar{J}_{\Delta}$, Taylor's formula gives

$$
v\left(X_{n}+t\left(X_{n+1}-X_{n}\right)\right)=v\left(X_{n}\right)+v^{\prime}\left(X_{n}\right) t\left(X_{n+1}-X_{n}\right)+\frac{v^{\prime \prime}\left(\xi_{n+1}\right)}{2} t^{2}\left(X_{n+1}-X_{n}\right)^{2},
$$

with $\left.\xi_{n+1} \in\right] \Delta, X_{n}[$. The convexity of $v$ implies

$$
0=v\left(X_{n}+t\left(X_{n+1}-X_{n}\right)\right) \geqslant v\left(X_{n}\right)+t \gamma_{n+1}\left(v^{\prime} b\right)\left(X_{n}\right)+t \sqrt{\gamma_{n+1}}\left(v^{\prime} \sigma\right)\left(X_{n}\right) U_{n+1} .
$$

Since $v^{\prime} b \geqslant 0$ on $\bar{J}_{\Delta}$ we have from (9)

$$
\begin{aligned}
A_{n+1} \cap\left\{X_{n} \in \bar{J}_{\Delta}\right\} & \subset\left\{\exists t \in[0,1], v\left(X_{n}\right)+t \sqrt{\gamma_{n+1}}\left(v^{\prime} \sigma\right)\left(X_{n}\right) U_{n+1} \leqslant 0\right\} \cap\left\{X_{n} \in \bar{J}_{\Delta}\right\}, \\
& \subset\left\{\exists t \in[0,1], t \sqrt{\gamma_{n+1}}\left|\left(v^{\prime} \sigma\right)\left(X_{n}\right) U_{n+1}\right| \geqslant v\left(X_{n}\right)\right\} \cap\left\{X_{n} \in \bar{J}_{\Delta}\right\} .
\end{aligned}
$$


Hence for any $n \geqslant 0$, we have on the event $\left\{X_{n} \in \bar{J}_{\Delta}\right\}$

$$
\begin{aligned}
\mathbf{P}\left[A_{n+1} \mid \mathcal{F}_{n}\right] & \leqslant \mathbf{P}\left[\left|U_{n+1}\right| \geqslant \frac{v\left(X_{n}\right)}{\sqrt{\gamma_{n+1}}\left|\left(v^{\prime} \sigma\right)\left(X_{n}\right)\right|} \mid \mathcal{F}_{n}\right] \\
& \leqslant \mathbf{P}\left[\left|U_{n+1}\right| \geqslant \frac{1}{c_{\sigma} \sqrt{\gamma_{n+1}}} \mid \mathcal{F}_{n}\right]
\end{aligned}
$$

by the domination assumption (8) on $v^{\prime} \sigma$. We conclude using property (5) of the random variable $U_{1}$.

Proof. By Lemma 2.2 we prove easily that for every $n \geqslant 0$

$$
\mathbf{P}\left[\Delta \in\left(X_{n}, X_{n+1}\right) \mid \mathcal{F}_{n}\right] \leqslant \exp \left(-\frac{1}{c_{\sigma}^{2} \gamma_{n+1}}\right) \quad \text { on } X_{n} \in \mathcal{U}_{\Delta}
$$

We now consider the event $\left\{X_{n} \notin \mathcal{U}_{\Delta}\right\}$. Then we have

$$
\begin{aligned}
\left\{\Delta \in\left(X_{n}, X_{n+1}\right)\right\} & =\left\{\exists t \in[0,1], U_{n+1}=\frac{\Delta-X_{n}}{t \sqrt{\gamma_{n+1}} \sigma\left(X_{n}\right)}-\sqrt{\gamma_{n+1}} \frac{b\left(X_{n}\right)}{\sigma\left(X_{n}\right)}\right\}, \\
& \subset\left\{\left|U_{n+1}\right| \geqslant \frac{\left|\Delta-X_{n}\right|}{\sqrt{\gamma_{n+1}}\left|\sigma\left(X_{n}\right)\right|}-\sqrt{\gamma_{n+1}} \frac{\left|b\left(X_{n}\right)\right|}{\left|\sigma\left(X_{n}\right)\right|}\right\} .
\end{aligned}
$$

As the drift $b$ is dominated by $C_{b}(1+|x|)$, we have

$$
\left\{\Delta \in\left(X_{n}, X_{n+1}\right)\right\} \subset\left\{\left|U_{n+1}\right| \geqslant\left(\frac{\left|\Delta-X_{n}\right|}{\sqrt{\gamma_{n+1}} C_{b}\left(1+\left|X_{n}\right|\right)}-\sqrt{\gamma_{n+1}}\right) \frac{C_{b}\left(1+\left|X_{n}\right|\right)}{\left|\sigma\left(X_{n}\right)\right|}\right\},
$$

and using the triangular inequality and $\left|X_{n}-\Delta\right| \geqslant \varepsilon$ we prove that $\frac{\left|\Delta-X_{n}\right|}{1+\left|X_{n}\right|} \geqslant \frac{1}{1+\frac{1+\mid \Delta}{\varepsilon}}$. We also deduce that there exists $n_{1} \geqslant 0$ and $C>0$ such that for any $n \geqslant n_{1}$,

$$
\begin{aligned}
\mathbf{P}\left[\left\{\Delta \in\left(X_{n}, X_{n+1}\right)\right\} \cap\left\{X_{n} \notin \mathcal{U}_{\Delta}\right\} \mid \mathcal{F}_{n}\right] & \leqslant \mathbf{P}\left[\left\{\left|U_{n+1}\right| \geqslant \frac{C}{\sqrt{\gamma_{n+1}}} \frac{C_{b}\left(1+\left|X_{n}\right|\right)}{\left|\sigma\left(X_{n}\right)\right|}\right\} \cap\left\{X_{n} \notin \mathcal{U}_{\Delta}\right\} \mid \mathcal{F}_{n}\right] \\
& \leqslant \mathbf{P}\left[\left\{\left|U_{n+1}\right| \geqslant \frac{C C_{b}}{C_{\sigma \sqrt{\gamma_{n+1}}}}\right\} \cap\left\{X_{n} \notin \mathcal{U}_{\Delta}\right\} \mid \mathcal{F}_{n}\right]
\end{aligned}
$$

using $|\sigma| \leqslant \sqrt{C_{\sigma}} \sqrt{V}$.

From (10) and (11) combined with (5), we get

$$
\exists n_{1} \geqslant 0, \exists C>0, \forall n \geqslant n_{1}, \quad \mathbf{P}\left[\Delta \in\left(X_{n}, X_{n+1}\right) \mid \mathcal{F}_{n}\right] \leqslant \exp \left(-\frac{C}{\gamma_{n+1}}\right) .
$$

By the condition on the sequence $\left(\gamma_{n}\right)_{n \geqslant 1}$ and the conditional Borel-cantelli lemma we deduce that the event $\left\{\Delta \in\left(X_{n}, X_{n+1}\right)\right\}$ occurs a finite number of times.

Remark 2.3. The condition on the step sequence $\left(\gamma_{n}\right)_{n \geqslant 1}$ is not restrictive. Indeed, it is satisfied for $\left(\gamma_{n}\right)_{n \geqslant 1}$ defined by $\gamma_{n}=\gamma_{0} n^{-r}$ with $\gamma_{0}>0$ and $\left.\left.r \in\right] 0,1\right]$, or $\gamma_{n}=\log (n)^{-r}$ with $r>1$.

The technical assumption " $v$ convex" is not very restrictive in practice. The important point to note is the condition $v^{\prime} b \geqslant 0$ which implies that $\Delta$ is unstable for the ODE $u^{\prime}=b(u)$. But $\Delta$ may be repulsive as well as attractive for the $\operatorname{SDE}\left(c f\right.$. Rem. 1.3). The condition $v^{\prime} \sigma=\mathcal{O}(v)$ in a neighborhood of $\Delta$ is very important and it seems difficult to relax it. 
Remark 2.4. It is easy to extend the above theorem to the case of finitely many boundary points $\Delta_{i}$. If for every $\Delta_{i}$, there exists a neighborhood $\mathcal{U}_{\Delta_{i}}$ and a convex function $v_{i} \in \mathcal{C}^{2}\left(\mathcal{U}_{\Delta_{i}}, \mathbf{R}\right)$ such that $v(\Delta)=0$ and satisfying (7), then

with $\Delta_{0}=-\infty$ and $\Delta_{l+1}=+\infty$.

$$
\mathbf{P}\left[\exists i \in\{0, \ldots, l\}, \exists n_{0} \geqslant 0, \forall n \geqslant n_{0}, X_{n} \in\right] \Delta_{i}, \Delta_{i+1}[]=1,
$$

\subsection{Weighted empirical measures}

Let $\left(\eta_{n}\right)_{n \geqslant 1}$ a positive sequence, called weight sequence, such that $H_{n}=\sum_{k=1}^{n} \eta_{k}$ increases to $+\infty$ when $n$ tends to $+\infty$. We define the weighted empirical measures $\left(\nu_{n}^{\eta}\right)_{n \geqslant 1}$ by

$$
\forall n \geqslant 1, \quad \nu_{n}^{\eta}(\mathrm{d} x)=\frac{1}{H_{k}} \sum_{k=1}^{n} \eta_{k} \delta_{X_{k-1}} .
$$

In this section we assume that the diffusion satisfies a stability condition i.e.

$$
\exists \alpha>0, \forall|x| \geqslant M, \quad x b(x)+\frac{1}{2} \sigma^{2}(x) \leqslant-\alpha x^{2} .
$$

This condition implies that the points $-\infty$ and $+\infty$ are strongly repulsive and that the empirical measures $\left(\nu_{t}\right)_{t \geqslant 0}$ are tight. Since $b$ and $\sigma$ have sublinear growth, this condition implies also the tightness of the weighted empirical measures $\left(\nu_{n}^{\eta}\right)_{n \geqslant 1}$ of the scheme and that any weak limit is an invariant probability for the diffusion (cf. [5] or [6]).

A consequence of Theorem 2.1 is the following proposition which describe the convergence of $\left(\nu_{n}^{\eta}\right)_{n \geqslant 1}$ according to the behavior of $b$ and $\sigma$ in a neighborhood of $\Delta$. For more clearness, we parametrize $b$ and $\sigma$.

Proposition 2.5. Let $\Delta$ the unique point of $\mathbf{R}$ such that $b(\Delta)=\sigma(\Delta)=0$. We assume that in a neighborhood of $\Delta$ we have $b(x)=\operatorname{sgn}(x-\Delta) \rho_{b}(x)$ and $\sigma(x)=\rho_{\sigma}(x)$ with $\rho_{b} \geqslant 0$,

$$
\rho_{b}(x) \sim c_{b}|x-\Delta|^{\beta} \quad \text { and } \quad \sigma(x) \sim c_{\sigma}|x-\Delta|^{\varsigma},
$$

where $\beta, \varsigma, c_{b}$ and $c_{\sigma}$ are positive real numbers and $\varsigma \geqslant 1$. If the step sequence $\left(\gamma_{n}\right)_{n \geqslant 1}$ satisfies $\forall C>0$, $\sum_{n \geqslant 1} \exp \left(-C / \gamma_{n}\right)<+\infty$, then

- if $1+\beta-2 \varsigma>0$ then $\Delta$ is an attractive boundary point and $\nu_{n}^{\eta} \Rightarrow \delta_{\Delta}$,

- if $1+\beta-2 \varsigma=0$ and $c_{\sigma}>\sqrt{2 c_{b}}$ then $\Delta$ is an attractive boundary point and $\nu_{n}^{\eta} \Rightarrow \delta_{\Delta}$,

- if $1+\beta-2 \varsigma=0, c_{\sigma}<\sqrt{2 c_{b}}$ and $\beta=1$ (which implies $\varsigma=1$ ) then $\Delta$ is a strongly repulsive boundary point and $\nu_{n}^{\eta} \Rightarrow \nu_{+}$or $\nu_{n}^{\eta} \Rightarrow \nu_{-}$,

- if $1+\beta-2 \varsigma<0, c_{\sigma} \leqslant \sqrt{2 c_{b}}$ and $\left.\left.\beta \in\right] 0,1\right]$ then $\Delta$ is a strongly repulsive boundary point and $\nu_{n}^{\eta} \Rightarrow \nu_{+}$ or $\nu_{n}^{\eta} \Rightarrow \nu_{-}$,

where $\nu_{+}$is the invariant probability on $] \Delta,+\infty\left[\right.$ and $\nu_{-}$the invariant probability on $]-\infty, \Delta[$.

Proof. To simplify notation, we assume without loss of generality that $\Delta=0$.

Let $\left.\mathcal{U}_{\Delta}=\right] \Delta-\varepsilon, \Delta+\varepsilon\left[\right.$ a neighborhood of $\Delta$ and the convex function $v(x)=x^{2}$. Then for every $x \in \mathcal{U}_{\Delta}$ we have

$$
v^{\prime} b=2 x \operatorname{sgn}(x) \rho_{b}(x) \geqslant 0 .
$$

Moreover $v^{\prime} \sigma \sim c_{\sigma}|x|^{\varsigma+1}$ with $\varsigma \geqslant 1$ hence there exists $C>0$ such that $\left|v^{\prime} \sigma\right| \leqslant C v$. By Theorem 2.1 we know then that the scheme lives in $]-\infty, \Delta[$ or in $] \Delta,+\infty[$ after an almost-surely finite random time.

Furthermore, with $v(x)=x^{2}$ we have

$$
\forall x \in \mathcal{U}_{\Delta}, \quad \mathcal{A} v(x)=2|x| \rho_{b}(x)+\sigma^{2}(x)=2 c_{b}|x|^{1+\beta}+c_{\sigma}^{2}|x|^{2 \varsigma}+o\left(|x|^{(1+\beta) \vee(2 \varsigma)}\right),
$$


and $\frac{1}{2} \sigma^{2}(x) \frac{\left(v^{\prime}(x)\right)^{2}}{v(x)}=2 \sigma^{2}(x) \sim 2 c_{\sigma}^{2}|x|^{2 \varsigma}$.

- If $1+\beta>2 \varsigma$, there exists a neighborhood of 0 in which $\mathcal{A} v<\frac{1}{2} \sigma \frac{\left(v^{\prime}\right)^{2}}{v}$. Hence by Proposition 1.2, 0 is an attractive boundary point. The sequence of weighted empirical measures $\left(\nu_{n}^{\eta}\right)_{n \geqslant 1}$ of the scheme is tight on $[0,+\infty[$ and on $]-\infty, 0]$, and any weak limit is an invariant probability. However, $\delta_{0}$ (the Dirac at 0 ) is the unique invariant probability on $[0,+\infty[$ or on $]-\infty, 0]$. Thus any weak limit of $\left(\nu_{n}^{\eta}\right)_{n \geqslant 1}$ is $\delta_{0}$, which proves the first item.

- If $1+\beta=2 \varsigma, \mathcal{A} v(x)=\left(2 c_{b}+c_{\sigma}^{2}\right)|x|^{2 \varsigma}+o\left(|x|^{2 \varsigma}\right)$. If $c_{\sigma}>\sqrt{2 c_{b}}$ there exists a neighborhood of 0 in which $\mathcal{A} v<2 \sigma^{2}$ and we conclude as above.

If $\beta=1$ and $c_{b}<\sqrt{2 c_{b}}$, we have for every $x$ in $\mathcal{U}_{\Delta}, \mathcal{A} v(x) \geqslant 2 \sigma^{2}(x)+\varepsilon x^{2}$. By Proposition 1.2, the point 0 is then strongly repulsive. Any weak limit of $\left(\nu_{n}^{\eta}\right)_{n \geqslant 0}$ is a probability on ] $-\infty, 0[$ or on $] 0,+\infty$ [, therefore we have $\nu_{n}^{\eta} \Rightarrow \nu_{-}$or $\nu_{n}^{\eta} \Rightarrow \nu_{+}$(we recall that the boundary points $-\infty$ and $+\infty$ are strongly repulsive).

- The proof for the case $1+\beta<2 \varsigma, c_{\varsigma} \leqslant \sqrt{2 c_{b}}$ and $\left.\left.\beta \in\right] 0,1\right]$ is similar.

In order to illustrate this result, let us consider the following example.

Example 2.6. We consider, like in [7], the function $V: \mathbf{R} \rightarrow \mathbf{R}_{+}$defined by

$$
V(x)=\left\{\begin{array}{ll}
(x-3 \operatorname{sgn}(x))^{2} & \text { if }|x| \geqslant 3, \\
\frac{1}{72}\left(x^{2}-9\right)^{2} & \text { if }|x| \leqslant 3
\end{array} \text { and } \quad b(x)=\left\{\begin{array}{ll}
-2(x-3 \operatorname{sgn}(x)) & \text { if }|x| \geqslant 3, \\
-\frac{1}{18} x^{3}+\frac{1}{2} x & \text { if }|x| \leqslant 3
\end{array},\right.\right.
$$

and $b=-V^{\prime}$. The ordinary differential equation $u^{\prime}=b(u)$ has 3 critical points: $-3,0$ and 3 . The points -3 and 3 are stable and the point 0 is unstable. Let $c \in] 0,2[$ a parameter and $\sigma$ defined by $\sigma(x)=c x$. We consider the process $\left(X_{t}\right)_{t \geqslant 0}$ solution of the SDE $\mathrm{d} X_{t}=b\left(X_{t}\right) \mathrm{d} t+\sigma\left(X_{t}\right) \mathrm{d} B_{t}$. It is clear that the point 0 is a boundary point for $\left(X_{t}\right)_{t \geqslant 0}$. Moreover we check that

$$
\mathcal{A} V(x)=-\left(4-c^{2}\right) x^{2}+12 \operatorname{sgn}(x) \quad \text { if }|x| \geqslant 3,
$$

and that the points $-\infty$ and $+\infty$ are then strongly repulsive.

On the other hand, we use Proposition 1.2 to determine the nature of the boundary point 0 according to $c$. Assume that $I=] 0,+\infty\left[\right.$ and let $v$ the function defined on $\left[0,+\infty\left[\right.\right.$ by $v(x)=x^{2}$. We have

$$
\forall x \in] 0,3\left[, \quad \mathcal{A} v(x)=\left(1+c^{2}\right) x^{2}-\frac{1}{9} x^{4} \quad \text { and } \quad \frac{1}{2} \sigma^{2}(x) \frac{\left(v^{\prime}(x)\right)^{2}}{v(x)}=2 c^{2} x^{2},\right.
$$

and then

- if $c<1$ the boundary point 0 is strongly repulsive (for $] 0,+\infty[$ and $]-\infty, 0[$ by symmetry). Indeed the condition (3) is satisfied with $\varepsilon=\frac{1-c^{2}}{2}$ and $\left.J_{\Delta}=\right] 0,3 \sqrt{\frac{1-c^{2}}{2}}[$;

- if $c>1$ it is easy to check that the boundary point 0 is attractive;

- if $c=1$ we consider the function $v(x)=x \exp (x)$ and we check that 0 is a repulsive boundary point.

Thus the nature of the boundary point 0 (which is always stable for the ODE $u^{\prime}=b(u)$ ) may change according to $c$. By Theorem 1.1 the ergodic behavior of $\left(X_{t}\right)_{t \geqslant 0}$ is the following:

- if $c \in] 1,2\left[\right.$ then $X_{t} \stackrel{a . s}{\longrightarrow} 0$ (for every starting point $X_{0}$ );

- if $c=1$ then $\frac{1}{t} \int_{0}^{t} \delta_{X_{s}}(\mathrm{~d} x) \Rightarrow \delta_{0}$; 


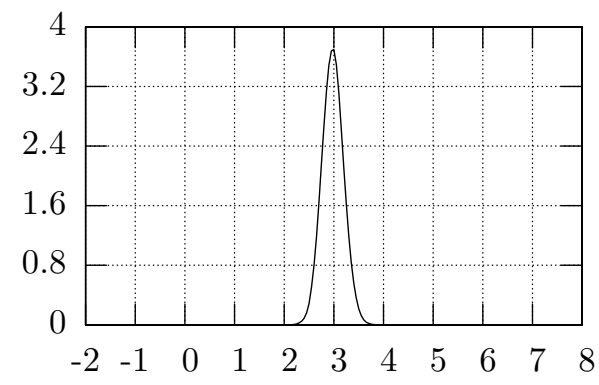

(a) $c=0.1$

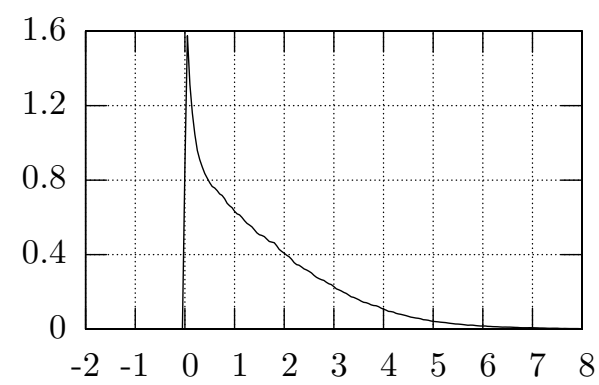

(c) $c=0.75$

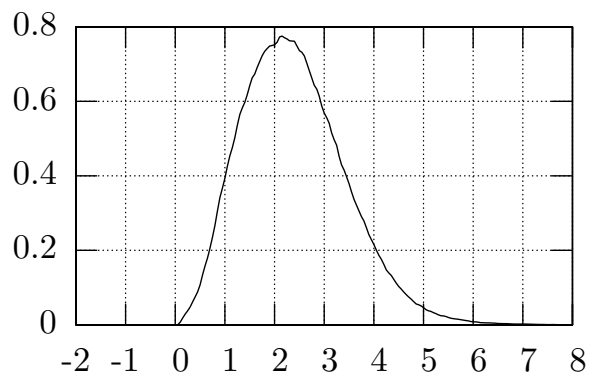

(b) $c=0.5$

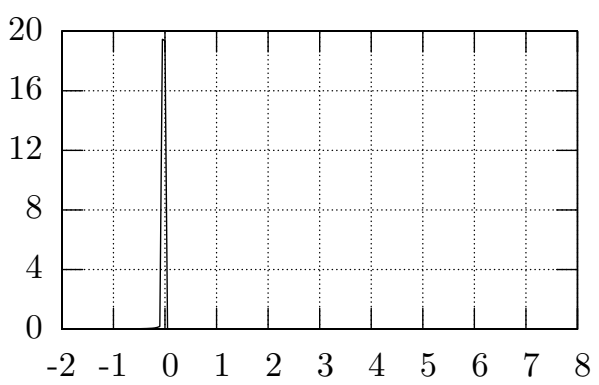

(d) $c=1$

FiguRE 1. Approximation of the stationary density for different values of $c$.

- if $c<1$ then for every $f \in \mathbf{L}^{1}(m)$

$$
\frac{1}{t} \int_{0}^{t} f\left(X_{s}\right) \mathrm{d} s \stackrel{a . s}{\longrightarrow} \begin{cases}\int f \mathrm{~d} \nu_{-} & \text {if } \left.X_{0} \in\right] 0,+\infty[ \\ f(0) & \text { if } X_{0}=0 \\ \int f \mathrm{~d} \nu_{+} & \text {if } \left.X_{0} \in\right]-\infty, 0[\end{cases}
$$

with $\nu_{-}$the invariant measure of $\left(X_{t}\right)_{t \geqslant 0}$ on $]-\infty, 0\left[\right.$ and $\nu_{+}$the invariant measure on $] 0,+\infty[$.

By the above Proposition, we know that the weighted empirical measure $\left(\nu_{n}^{\eta}\right)_{n \geqslant 1}$ weakly converge to $\delta_{0}$ when $c \in] 1,2\left[\right.$ and to $\nu_{+}$or $\nu_{-}$when $\left.c \in\right] 0,1\left[\right.$. Note that the convergence to $\nu_{+}$or $\nu_{-}$does not depend on the initial condition and is not previsible.

We give a representation of the density of $\nu$ approximated by $\nu_{n}^{\eta}$ with $n=10^{6}$. More precisely, we discretize the interval $[-2,8]$ using 200 intervals $I_{i}$ of length 0.05 and we compute $\nu_{n}^{\eta}\left(\mathbf{1}_{I_{i}}\right)$ for each $I_{i}$ with $n=10^{6}$. The step sequence $\left(\gamma_{n}\right)_{n \geqslant 1}$ is defined by $\gamma_{n}=n^{-1 / 3}$ and the weight sequence $\left(\eta_{n}\right)_{n \geqslant 1}$ is defined by $\eta_{n}=1$. The results of this approximation of the stationary density are given in Figure 1 for different values of $c$.

We remark that for a small noise $(c=0.1)$, the invariant probability concentrates around a stable point of the ODE $u^{\prime}=b(u)$ (the point 3), and the more coefficient of diffusion increases, the more the invariant measure is spread out. For $c=0.75$ we show that the invariant measure is infinite at 0 , and for $c=1$ the invariant measures seems to be the Dirac mass at 0 . 


\section{A. Proof of Theorem 1.1}

We first prove the following lemma.

Lemma A.1. If the two boundary points $l$ and $r$ are repulsive then the diffusion is positive recurrent if and only if its speed measure is finite.

Proof. By definition, the diffusion is positive recurrent if and only if for all $a$ and $b$ in $I, \mathbf{E}_{a}\left[T_{b}\right]<+\infty$ and $\mathbf{E}_{b}\left[T_{a}\right]<+\infty$. Let $l<a<b<r$. By symmetry it is sufficient to prove that

$$
\mathbf{E}_{a}\left[T_{b}\right]<+\infty \quad \Leftrightarrow \quad \int_{l}^{a} m(y) \mathrm{d} y<+\infty
$$

Firstly, $l$ is repulsive and $T_{l}=\lim _{x \rightarrow l} T_{x}$ therefore $\mathbf{E}_{a}\left[T_{b}\right]=\mathbf{E}_{a}\left[T_{b} \wedge T_{l}\right]=\lim _{x \rightarrow l} \mathbf{E}_{a}\left[T_{b} \wedge T_{x}\right]$. Moreover we have $\forall x \in] l, a[$,

$$
\mathbf{E}_{a}\left[T_{b} \wedge T_{x}\right]=\mathbf{P}_{a}\left[T_{b}<T_{x}\right] \int_{a}^{b}(p(b)-p(y)) m(y) \mathrm{d} y+\mathbf{P}_{a}\left[T_{x} \leqslant T_{b}\right] \int_{x}^{a}(p(y)-p(x)) m(y) \mathrm{d} y,
$$

and since $\int_{a}^{b}(p(b)-p(y)) m(y) \mathrm{d} y$ is finite and does not depend on $x$, the limit when $x$ tends to $l$ of $\mathbf{E}_{a}\left[T_{b} \wedge T_{x}\right]$ is finite if and only if

$$
\lim _{x \rightarrow l}\left(\mathbf{P}_{a}\left[T_{x} \leqslant T_{b}\right] \int_{x}^{a}(p(y)-p(x)) m(y) \mathrm{d} y\right)<+\infty .
$$

As $\mathbf{P}_{a}\left[T_{x} \leqslant T_{b}\right]=\frac{p(b)-p(a)}{p(b)-p(x)}$ we have

$$
\begin{aligned}
\mathbf{P}_{a}\left[T_{x} \leqslant T_{b}\right] \int_{x}^{a}(p(y)-p(x)) m(y) \mathrm{d} y & =(p(b)-p(a)) \int_{x}^{a} \frac{p(y)-p(x)}{p(b)-p(x)} m(y) \mathrm{d} y \\
& =(p(b)-p(a)) \int_{x}^{a} \mathbf{P}_{y}\left[T_{b}<T_{x}\right] m(y) \mathrm{d} y
\end{aligned}
$$

and it follows that $\mathbf{E}_{a}\left[T_{b}\right]<+\infty$ if and only if $\lim _{x \rightarrow l} \int_{x}^{a} \mathbf{P}_{y}\left[T_{b}<T_{x}\right] m(y) \mathrm{d} y<+\infty$. Since $T_{x}$ strictly increases to $T_{l}, \mathbf{P}_{y}\left[T_{b}<T_{x}\right]$ increases to $\mathbf{P}_{y}\left[T_{b}<T_{l}\right]=1$ because $l$ is repulsive. The monotone convergence theorem yields (15).

Proof of Theorem 1.1. The first two items are proved in [3] (Prop. 5.22). We prove the third item. Suppose that $l$ and $r$ are repulsive. By definition of the scale function we have for all $l<a<x<b<r$

$$
\mathbf{P}_{x}\left[\inf _{0 \leqslant t<\zeta} X_{t} \leqslant a\right] \geqslant \mathbf{P}_{x}\left[x_{T_{a} \wedge T_{b}}=a\right]=\frac{p(b)-p(x)}{p(b)-p(a)}
$$

Increasing $b$ to $r$ we obtain $\mathbf{P}_{x}\left[\inf _{0 \leqslant t<\zeta} X_{t} \leqslant a\right]=1$ since $r$ is repulsive. The limit when $a$ decreases to $l$ also gives

$$
\mathbf{P}_{x}\left[\inf _{0 \leqslant t<\zeta} X_{t}=l\right]=1 .
$$

In the same way we obtain $\mathbf{P}_{x}\left[\sup _{0 \leqslant t<\zeta} X_{t}=r\right]=1$. The diffusion is thus recurrent on $I$ and $\zeta=+\infty$ a.s. Moreover, by Lemma A.1 we know that the recurrence is positive if and only if the speed measure is finite.

- If the two boundary points are repulsive then the speed measure is finite and by Theorem (53.1) in [8] we have

$$
\forall f \in \mathbf{L}^{1}(\nu), \quad \frac{1}{t} \int_{0}^{t} f\left(X_{s}\right) \mathrm{d} s \Rightarrow \int_{\mathbf{R}} f \mathrm{~d} \nu \quad \text { a.s. }
$$

where $\nu$ is the normalized speed measure. 
- If $l$ is strongly repulsive and $r$ is repulsive then the diffusion is null recurrent. Considering an increasing sequence of continuous functions with compact support $\left(g_{n}(x)\right)_{n \geqslant 1}$ such that $g_{n}(x) \rightarrow 1$ and $\forall n \geqslant 1$, $\int_{\mathbf{R}} g_{n}(x) m(\mathrm{~d} x) \neq 0$, we obtain by Theorem (53.1) in [8]

$$
\forall f \in \mathbf{L}^{1}(m), \quad \frac{1}{t} \int_{0}^{t} f\left(X_{s}\right) \mathrm{d} s \stackrel{a . s}{\longrightarrow} 0 .
$$

On the other hand, we consider a sub-sequence $\left(\nu_{a(t)}\right)_{t \geqslant 0}$ of $\left(\nu_{t}\right)_{t \geqslant 0}$ converging to a measure $\nu$ (the empirical measures are tight). Let $f$ be a continuous function with compact support such that $\operatorname{supp}(f) \subset$ $\left[l, r\left[\right.\right.$. As $\nu_{a(t)} \Rightarrow \nu$ we have

$$
\frac{1}{a(t)} \int_{0}^{a(t)} f\left(X_{s}\right) \mathrm{d} s \stackrel{a . s}{\longrightarrow} \int f \mathrm{~d} \nu
$$

The boundary point $l$ is strongly repulsive and $\operatorname{supp}(f) \subset[l, r[$, thus $f$ is integrable with respect to $m$. Hence (16) implies $\int f \mathrm{~d} \nu=0$. The interval $[l, r[$ satisfies

$$
\forall f \in \mathcal{C}_{c}(\bar{I}), \operatorname{supp}(f) \subset[l, r[\Rightarrow \nu(f)=0,
$$

therefore $\operatorname{supp}(\nu)=\{r\}$. Since $\nu$ is normalized we have $\nu=\delta_{r}$.

- In the same way, if the two boundary points are strongly repulsive then any weak limit of $\left(\nu_{t}\right)_{t \geqslant 0}$ is a measure with support $\{l, r\}$.

\section{B. Proof of Proposition 1.2}

We first prove the following proposition which is equivalent to the Proposition 1.2.

Proposition B.1. Let $\Delta$ be a boundary point (finite or infinite, left endpoint or right endpoint) of I. The following statements are equivalents

(1) $\Delta$ is a repulsive boundary point of $I$ if and only if there exists a neighborhood $J_{\Delta} \subset I$ of $\Delta$ and a strictly monotone function $V \in \mathcal{C}^{2}\left(J_{\Delta}, \mathbf{R}_{+}\right)$such that

$$
\lim _{x \rightarrow \Delta} V(x)=+\infty \quad \text { and } \quad \forall x \in J_{\Delta}, \quad \mathcal{A} V(x) \leqslant 0 .
$$

(2) $\Delta$ is a strongly repulsive boundary point of $I$ if and only if there exists a neighborhood $J_{\Delta} \subset I$ of $\Delta$ and a strictly monotone function $V \in \mathcal{C}^{2}\left(J_{\Delta}, \mathbf{R}_{+}\right)$such that

$$
\exists \varepsilon>0, \forall x \in J_{\Delta}, \quad \mathcal{A} V(x) \leqslant-\varepsilon .
$$

(3) $\Delta$ is a attractive point of $I$ if and only if there exists a neighborhood $J_{\Delta} \subset I$ of $\Delta$ and a strictly monotone function $V \in \mathcal{C}^{2}\left(J_{\Delta}, \mathbf{R}_{+}\right)$such that

$$
\sup _{x \in J_{\Delta}} V(x)=V(\Delta)<+\infty \quad \text { and } \quad \forall x \in J_{\Delta}, \quad \mathcal{A} V(x) \geqslant 0 .
$$

Proof. We give the proof when $\Delta$ is a right endpoint of $I$. Then $J_{\Delta}$ is an interval $] c, \Delta[$ with $c \in I$.

- - We suppose that there exists a neighborhood $J_{\Delta}$ of $\Delta$ and a function $V \in \mathcal{C}^{2}\left(J_{\Delta}, \mathbf{R}_{+}\right)$such that $\lim _{x \rightarrow \Delta} V(x)=+\infty$ and $\mathcal{A} V \leqslant 0$ on $J_{\Delta}$. For every $x \in J_{\Delta}$ we have

$$
\mathcal{A} V(x)=\frac{1}{m(x)}\left(\frac{V^{\prime}(x)}{p^{\prime}(x)}\right)^{\prime} \leqslant 0
$$


hence $V^{\prime} / p^{\prime}$ is decreasing on $J_{\Delta}$. There also exists $C>0$ such that $V^{\prime}(x) \leqslant C p^{\prime}(x)$ for every $\left.x \in\right] c, \Delta[$ since $p^{\prime}>0$. It follows that $\lim _{x \rightarrow \Delta} p(x)-p(c)=+\infty$ because $V$ tends to infinity in $\Delta$.

- Conversely we must find the good Lyapunov function $V$. Let $c>0$ be such that $p(c)>0$ (c exists because $\Delta$ is repulsive). Since $p$ is strictly increasing we have for every $x \in] c, \Delta[, p(x)>p(c)>0$. We also define the function $V$ on $] c, \Delta[$ by

$$
\forall x \in] c, \Delta[, \quad V(x)=p(x)-p(c) .
$$

The point $\Delta$ is repulsive thus $V$ increases to infinity when $x$ tends to $\Delta$. Moreover $V \in \mathcal{C}^{2}(] c, \Delta\left[, \mathbf{R}_{+}\right)$ and $\mathcal{A} V=0$.

- - Let $V \in \mathcal{C}^{2}\left(J_{\Delta}, \mathbf{R}_{+}\right)$be such that $\lim _{x \rightarrow \Delta} V(x)=+\infty$ and $\varepsilon>0$ such that $\mathcal{A} V \leqslant-\varepsilon$. Thus we have

$$
\int_{J_{\Delta}} \mathcal{A} V(x) m(x) \mathrm{d} x \leqslant-\varepsilon \int_{J_{\Delta}} m(x) \mathrm{d} x
$$

and since $\mathcal{A} V(x)=\frac{1}{m(x)}\left(\frac{V^{\prime}(x)}{p^{\prime}(x)}\right)^{\prime}$ we obtain for every $c \in J_{\Delta}$,

$$
\int_{c}^{\Delta} \mathcal{A} V(x) m(x) \mathrm{d} x=\lim _{x \rightarrow \Delta} \frac{V^{\prime}(x)}{p^{\prime}(x)}-\frac{V^{\prime}(c)}{p^{\prime}(c)} .
$$

By (17) we derive that

$$
\int_{c}^{\Delta} m(x) \mathrm{d} x \leqslant \frac{1}{\varepsilon}\left(\frac{V^{\prime}(c)}{p^{\prime}(c)}-\lim _{x \rightarrow \Delta} \frac{V^{\prime}(x)}{p^{\prime}(x)}\right)
$$

As the functions $V$ and $p$ are increasing on $J_{\Delta}$ we have $\lim _{x \rightarrow \Delta} \frac{V^{\prime}(x)}{p^{\prime}(x)} \geqslant 0$, which gives $\int_{c}^{\Delta} m(x) \mathrm{d} x \leqslant C$ (i.e. $\Delta$ strongly repulsive).

- Conversely we assume that $\Delta$ is strongly repulsive. Let $c \in I$ and $V$ the function defined on $] c, \Delta[$ by

$$
\forall x \in] c, \Delta\left[, \quad V(x)=\int_{c}^{x}\left(p^{\prime}(y) \int_{y}^{\Delta} m(z) \mathrm{d} z\right) \mathrm{d} y\right.
$$

It is clear that $V \in \mathcal{C}^{2}(] c, \Delta\left[, \mathbf{R}_{+}\right)$and that for every $\left.x \in\right] c, \Delta\left[, V^{\prime}(x)=p^{\prime}(x) \int_{x}^{\Delta} m(z) \mathrm{d} z\right.$. Moreover

$$
\forall x \in] x, \Delta[, \quad \mathcal{A} V(x)=-1 .
$$

- We prove (3) in the same manner as (1). For the converse we consider the function $V(x)=p(x)-p(c)$ on $] c, \Delta[$ with $c$ such that $p(c)>0$.

Proof of Proposition 1.2. Let $J_{\Delta}$ a neighborhood of $\Delta$ strictly included in $I$. We consider the case in which $\Delta$ is the right endpoint of $I$ i.e. $\left.J_{\Delta}=\right] c, \Delta\left[\right.$ with $c \in I$. We first define for $L \geqslant 0$ the function $\phi_{L}$ by

$$
\begin{aligned}
\phi_{L}:[0, \exp (L)[ & \rightarrow \mathbf{R}_{+}, \\
x & \mapsto-\ln (x)+L .
\end{aligned}
$$


The function $\phi_{L}$ is a strictly decreasing one-to-one $\mathcal{C}^{\infty}$ function. Moreover, for every $\mathcal{C}^{2}$ function $v$ with values in $[0, \exp (L)[$ we have

$$
\mathcal{A}\left(\phi_{L} \circ v\right)=-\frac{\mathcal{A} v}{v}+\frac{1}{2} \sigma^{2} \frac{\left(v^{\prime}\right)^{2}}{v^{2}}
$$

- - We assume that there exists $v \in \mathcal{C}^{2}\left([c, \Delta], \mathbf{R}_{+}\right)$strictly monotone satisfying $v(\Delta)=0$ and (2). We also define on $] c, \Delta[$ the function $V$ by $\forall x \in] c, \Delta\left[, V(x)=\left(\phi_{L} \circ v\right)(x)\right.$ with $L=\ln (v(c))$. It is a strictly monotone function which tends to infinity in $\Delta$. By (2) and (19) we obtain $\mathcal{A} V \leqslant 0$ on $] c, \Delta[$. The proposition (B.1) implies that $\Delta$ is repulsive.

- Conversely if $\Delta$ is repulsive then there exists $V \in \mathcal{C}^{2}(] c, \Delta\left[, \mathbf{R}_{+}\right)$strictly monotone which goes to $+\infty$ when $x$ tends to $\Delta$. We also define $v=\phi_{L}^{-1} \circ V$ on $] c, \Delta\left[\right.$ with $L=\inf _{x \in] c, \Delta[} V(x)$, and we extend it by continuity on $[c, \Delta]$ letting $v(c)=1$ and $v(\Delta)=0$. By $\mathcal{A} V \leqslant 0$ and (19) we have $\mathcal{A} v \geqslant \frac{1}{2} \sigma^{2} \frac{\left(v^{\prime}\right)^{2}}{v}$ on ]$c, \Delta[$.

- - Let $v$ a strictly monotone function on $[c, \Delta]$ such that $v(\Delta)=0$. For $\theta \geqslant 0$ and $L=\ln (v(c)+\theta)$ we define $V(x)=\left(\phi_{L, \theta} \circ v\right)(x)$ for every $\left.x \in\right] c, \Delta[$. This function $V$ is strictly monotone on $] c, \Delta[$ and admits a limit (finite or not) when $x$ tends to $\Delta$. From (3) and (19) we deduce that

$$
\forall x \in] c, \Delta[, \quad \mathcal{A} V(x) \leqslant-\varepsilon .
$$

- Conversely we consider the function $v=\phi_{L}^{-1} \circ V$ on $] c, \Delta\left[\right.$ with $L=\inf _{x \in] c, \Delta[} V(x)$ and we extend it by continuity letting $v(c)=1$ and $v(\Delta)=\lim _{x \rightarrow \Delta} \exp (-V(x))$.

- The proof is similar to the first two items.

\section{REFERENCES}

[1] W. Feller, The parabolic differential equations and the associated semi-groups of transformations. Ann. of Math. (2) 55 (1952) 468-519.

[2] W. Feller, Diffusion processes in one dimension. Trans. Amer. Math. Soc. 77 (1954) 1-31.

[3] I. Karatzas and S.E. Shreve, Brownian motion and stochastic calculus. Springer-Verlag, New York, 2nd edition, Graduate Texts in Mathematics 113 (1991).

[4] S. Karlin and H.M. Taylor, A second course in stochastic processes. Academic Press Inc. [Harcourt Brace Jovanovich Publishers], New York (1981).

[5] D. Lamberton and G. Pagès, Recursive computation of the invariant distribution of a diffusion. Bernoulli 8 (2002) $367-405$.

[6] V. Lemaire, Estimation récursive de la mesure invariante d'un processus de diffusion. Ph.D. Thesis, Université de Marne-laVallée (2005).

[7] G. Pagès, Sur quelques algorithmes récursifs pour les probabilités numériques. ESAIM Probab. Statist. 5 (2001) 141-170 (electronic).

[8] L.C.G. Rogers and D. Williams, Diffusions, Markov processes, and martingales. Vol. 1. Wiley Series in Probability and Mathematical Statistics: Probability and Mathematical Statistics. John Wiley \& Sons Ltd., Chichester, 2nd edition (1994).

[9] W.F. Stout, Almost sure convergence. Academic Press [A subsidiary of Harcourt Brace Jovanovich, Publishers], New YorkLondon, Probability and Mathematical Statistics 24 (1974). 